\title{
MIGRACION Y CALIDAD DE VIDA. UNA PROPUESTA DE ESTUDIO CENTRADA EN LOS MIGRANTES
}

\author{
Migration and quality of life. a study proposal focused on migrants
}

\author{
Roberta Panzeri \\ Fecha recepción: 12/07/2016 \\ Fecha aceptación: 11/11/2016
}

RESUMEN: La migración internacional es un fenómeno relevante que caracteriza el momento actual, en el que se ocultan muchas desigualdades. Este artículo examina las principales teorías explicativas presentes en la literatura sobre migración, las organiza y agrupa según la perspectiva que utilizan. Después de indicar las debilidades de cada enfoque, este trabajo subraya algunas grandes separaciones, que resaltan la necesidad de un análisis integrado que considere contemporáneamente los distintos niveles que constituyen este complejo fenómeno. Con esta finalidad se propone utilizar el "Capability Approach" como enfoque multidimensional para el análisis del bienestar y desarrollo humano de los migrantes. El propósito último de este artículo es demostrar la necesidad de involucrar a los propios migrantes, como agentes variados que se diferencian entre sí por una serie de características personales, y que deciden mudarse por una pluralidad de razones, además de estar respaldados por muy diversas condiciones políticas, sociales y económicas. Este trabajo pretende proporcionar la base teórica para una futura investigación empírica, que estudie la satisfacción de los migrantes, analice la influencia de la experiencia migratoria en el desarrollo y conjunto de capacidades de la persona, en término de realización personal percibida y logro de objetivos individuales.

PALABRAS CLAVE: Migración, Movilidad, Desarrollo, Bienestar, Capacidades.

ABSTRACT: International migration is an important phenomenon that characterizes the present day, in which are hidden many inequalities. This article examines the main explanatory theories found in the literature on migration, organized and grouped according to the perspective used. After indicating weaknesses of each approach, this paper highlights some large gaps, which highlight the need for an integrated analysis that considers simultaneously the different levels that make up this complex phenomenon. To this end it is proposed to use the "Capability Approach" as a multidimensional approach to analyzing welfare and human development of migrants. The ultimate purpose of this article is to demonstrate the need to involve migrants themselves, as distinctive agents that diverge from each other by a number of personal characteristics, who decide to move for a quantity of reasons, besides to be supported by very different political, social and economic backgrounds. This work aims to provide the theoretical basis for future empirical research to assess the satisfaction of migrants, analyze the influence of migratory experience in development and skill set of the person, in terms of perceived personal fulfillment and achievement of individual goals.

KEYWORDS: Migration, Mobility, Development, Wellbeing, Capabilities. 


\section{Introducción}

Las migraciones internacionales representan un rasgo distintivo de las dinámicas sociales del mundo contemporáneo. Se producen por muchas razones, conllevan diferentes escalas geográficas y pueden tener implicaciones políticas y económicas a nivel mundial.

Al mismo tiempo, representan hoy en día uno de los aspectos más controvertidos de la globalización (Bonfanti, 2014) sobre todo por dos razones: primeramente por la incoherencia entre las restrictivas condiciones de movilidad de las personas en comparación con la fácil circulación de bienes y capitales, y en segundo lugar por la multiplicidad y complejidad de características que pueden asumir las relaciones entre los migrantes y sus sociedades de origen y de acogida (de Haas y Rodríguez, 2010).

Para comprender de inmediato la magnitud del fenómeno en su conjunto, nos pueden venir en ayuda algunos datos. El número de migrantes internacionales mundiales aumentó más del doble entre 1980 y 2010, pasando de 103 millones a 220 millones, y ha seguido creciendo rápidamente en los últimos años. En 2013 llegó a 232 millones, un máximo histórico que ha sido superado en 2015 llegando a 244 millones (Department of Economic and Social Affairs, 2016) y se proyecta que esta tendencia seguirá, alcanzando más de 400 millones en 2050 (Martin, 2013).

Un fenómeno variado y complejo el de la migración, cuya trascendencia y capilaridad representan al mismo tiempo un desafío y una oportunidad para el desarrollo de las comunidades de ciudadanos y la mejora de las estructuras administrativas y legislativas de los países europeos, ya que la capacidad de gestionar la movilidad y la garantía de buenas condiciones de vida dentro del país se convierten en uno de los factores fundamentales para garantizar el éxito y la sostenibilidad de cada una de las naciones y de la Unión Europea en su conjunto.

Además, si hasta los años 50 los inmigrantes eran identificados siempre y sólo con gente de baja clase social que buscaba oportunidades de mejora económica en los países ricos, la situación actual es mucho más compleja: por ejemplo la existencia de medios de transporte legales asequibles ha dado la oportunidad a muchas más personas para viajar; también es cada vez más difusa la migración de personas con un alto nivel de estudio, procedentes de familias ricas, que migran en busca de mejores condiciones de vida, no siempre y no sólo a nivel económico; por último hay ahora una masiva ola migratoria por razones políticas o humanitarias. Esto hace que la población migrante sea más compleja y se vuelva difícil evaluar la satisfacción de los migrantes en el país de llegada. Hay indicios que nos llevan a pensar que pueda abrirse un escenario en el que los ciudadanos decidirán dónde vivir. Todo esto podría transformar totalmente los juegos: a los países debería interesarle cada vez más la satisfacción de sus ciudadanos, para tener la garantía de que éstos reiteren constantemente la elección de quedarse en el mismo país y no mudarse a otro que ofrece más oportunidades, un mayor nivel de calidad de vida, o más satisfacciones.

Los migrantes se convierten, aunque a veces temporalmente, en ciudadanos de nuestras sociedades y necesitaremos comprender los flujos migratorios y encontrar la mejor manera de manejar el fenómeno. Una importante pregunta entonces es si nuestras sociedades, en particular las europeas, que en este momento histórico juegan el papel de países anfitriones (en efecto o en potencia), poseen adecuadas herramientas de conocimiento para comprender el fenómeno migratorio que les afecta y la manera para que, tanto los migrantes como la sociedad acogedora, puedan beneficiarse de este dinamismo.

Lamentablemente, con frecuencia el discurso oficial de la política se centra de manera demagógica sólo en los efectos perjudiciales que la presencia de los migrantes tendría sobre 
los nativos, alimentando el miedo o el odio de la opinión pública generalista hacia algunas minorías señaladas como "enemigo público". Poco o nada de espacio se da a la comprensión de las condiciones que influyen en el (bien)estar de los migrantes, como sujetos y como actores del movimiento y de la transformación social. Esta brecha de conocimiento debe ser llenada, si queremos dar un salto cualitativo en nuestra capacidad de imaginar una manera de estar y convivir en una sociedad cada vez más mezclada. La alternativa a esta comprensión es la marginalización de ciertos sectores de la población, la fragmentación y segregación de la sociedad del futuro y su consecuente declive.

En el presente trabajo se pretende contribuir a la literatura sobre la migración internacional identificando y sistematizando los elementos claves que distintas corrientes de literatura han establecido como determinantes del fenómeno migratorio, es decir las causas y las razones que explicarían dicho fenómeno.

Se presentarán los tres principales ejes de abordaje del fenómeno migratorio: primero se afrontará el nivel macro, es decir las dinámicas internacionales que afectan las condiciones estructurales de los Estados implicados, ya que una amplia franja de la literatura se ha centrado en los macros factores que influencian la migración, entre los cuales las estructuras económicas, políticas y socio-culturales de los países de destino y de origen. Seguirá el nivel micro, ya que otra rama de la literatura se ha ocupado de las migraciones a partir del individuo como unidad de análisis. Por último se profundizará un tercer nivel de análisis, lo que se ha llamado nivel "meso" (Faist, 2009), que analiza en particular la función de las redes migratorias. Las redes sociales y familiares tienen un papel fundamental, ya que pueden, aunque sea indirectamente, condicionar e influenciar los procesos de transformación y de cambio de estas mismas estructuras.

Esta recopilación procura evaluar de manera crítica las teorías que se ocupan del proceso migratorio, subrayando las dificultades de los enfoques económicos neo-clásicos y de las perspectivas tradicionales, y presentando una lectura que pone de relieve las grandes separaciones que las caracterizan.

En segundo lugar, para intentar superar la parcialidad de estos ejes de estudio, se propondrá un marco teórico multidimensional que permita tener en cuenta varios aspectos, a parte del económico, para indagar y analizar el bienestar y desarrollo humano.

Y por último se sugiere la necesidad de abordar el estudio del fenómeno migratorio desde una perspectiva que tome en consideración el bienestar general de los migrantes en un sentido amplio y multidimensional, haciendo el esfuerzo de tener en cuenta su subjetividad, sin aplanar o simplificar su posición dentro de generalizaciones o abstracciones. Para conseguir esto es importante indagar su percepción subjetiva en la capacidad de transformar la migración en una ocasión para el aumento del desarrollo humano y el crecimiento del bienestar.

\section{La perspectiva Macro}

La perspectiva macro percibe la migración internacional como consecuencia de las diferencias de condiciones estructurales, demográficas, políticas y laborales de los territorios involucrados, y sobre todo en las diferencias geográficas en la demanda y la oferta de trabajo. Esta perspectiva se ve reflejada en los denominados push-pull models, donde aquellos conocidos como "demand-pull factors" son los factores característicos del país de acogida que atraerían a los migrantes, mientras que los llamados "supply-push factors" son los factores presentes en el país de origen (por ejemplo, salarios bajos o altas tasas de desempleo), que impulsarían la gente a desplazarse en búsqueda de oportunidades económicas. 
La que mejor encarna este pensamiento es la Teoría Neo-clásica, que fundamenta sus raíces teóricas en la perspectiva social funcionalista (Todaro, 1969) la cual considera la sociedad como un sistema con una tendencia hacia el equilibrio. Según este enfoque la migración pondría de relieve el importante papel desempeñado por las diferencias geográficas en la oferta y la demanda de mano de obra y subrayaría el papel de las diferencias en los salarios y las condiciones de empleo entre los países, ya que el diferencial económico de los salarios hace que los trabajadores del país de bajos salarios se muevan hacia países de altos salarios.

La Teoría del Conflicto ve a la sociedad como una entidad dinámica en constante proceso de cambio como consecuencia de la competición por los escasos recursos. Las perspectivas asociadas con la teoría del conflicto son:

La World System Theory (Wallerstein, 1980) argumenta que la migración internacional es un subproducto del capitalismo global, una consecuencia natural de alteraciones y trastornos que se producen en el proceso de desarrollo del capitalismo. La migración sería vista como un proceso que sirve a los intereses de las grandes corporaciones y de los grupos de intereses económicos específicos y de los Estados capitalistas que presionan por sus intereses.

La Teoría del Mercado del Trabajo Dual (Piore, 1972) sugiere que las economías desarrolladas son duales, es decir que tienen un mercado "primario" de trabajo seguro, bien remunerado, generalmente administrado por ciudadanos nacionales y un mercado "secundario" de empleo de baja remuneración y malas condiciones. La migración internacional sería impulsada principalmente por factores de atracción (empleo), ya que la segmentación de los mercados de trabajo crea una demanda permanente de mano de obra barata cubierta por los migrantes en la parte inferior del mercado de trabajo, es decir en el extremo "secundario", para ocupar aquellos puestos de trabajo que los trabajadores "primarios" normalmente evitan, sobre todo debido al estigma social asociado y a las malas condiciones.

\section{Limitaciones que caracterizan los enfoques Macro}

- Aunque hay consenso en que los macro factores contextuales económicos y políticos juegan "algún" papel, no hay acuerdo sobre qué tipo de papel desempeña cada uno de estos factores push-pull, su peso relativo y la interacción mutua entre ellos, ya que no se específica sus tareas, si no de manera muy vaga.

- Los modelos push-pull fallan en conceptualizar la migración como un proceso, y tienden por el contrario a ser estáticos. Debido a que la teoría subyacente explica que la sociedad es un sistema en busca de equilibrio, en teoría, una vez alcanzado el equilibrio, no debería darse la necesidad de más cambios. Eso supone que la migración podría algún día terminar.

- Tal vez el punto más importante es que los modelos macro no tienen en cuenta la voluntad del sujeto migrante, es decir su "agency" definida como la capacidad de las personas de tomar decisiones propias e independientes - de actuar o no actuar de una manera específica - y de alterar la estructura. El migrante figuraría como un peón pasivo en un juego cuyas reglas son decididas por otros. En realidad, sabemos que en la toma de decisión para migrar entran en juego, aunque de forma inconsciente, también factores personales, como la motivación, la capacidad de resolución de problemas, las competencias relacionales, las habilidades de comunicación, la capacidad de coping (tanto las capacidades reales, es decir experimentadas anteriormente, como las capacidades solamente percibidas). 
- Los modelos macro ignoran en gran medida aquellos conductores de migración que no son económicos y por lo general no logran explicar la migración impulsada por el aumento de un desarrollo que no sea monetario. Estos modelos no son suficientes para explicar los reales procesos de migración que tienen un fuerte y no aleatorio patrón, como por ejemplo la migración de retorno o la migración en ausencia de diferencia salarial (de Haas, 2011).

\section{La perspectiva Micro}

Las teorías que toman en consideración la perspectiva micro son aquellas que explican el fenómeno de la migración a partir del sujeto individual, es decir que identifican al individuo como unidad de análisis.

La Teoría Neo-clásica apoya una posición de conveniencia individualista y asumen que la decisión de un individuo de migrar es el resultado de un cálculo matemático y totalmente racional de costo-beneficio, con el único propósito de aumentar beneficio para sí mismo (self-interest). Este enfoque supone que cualquiera pueda disponer de libre elección y tenga completo acceso a la información.

La Teoría del Conflicto, a la inversa, considera los individuos como víctimas pasivas de las macro-fuerzas económicas. En particular, la teoría marxista utiliza el concepto de "falsa conciencia", que puede definirse como la falta de reconocimiento de los instrumentos de la propia opresión o explotación, para justificar el comportamiento de una clase oprimida, es decir de los migrantes, que involuntariamente adopta los mismos puntos de vista de la clase opresora (de Haas, 2011).

\section{Limitaciones que caracterizan los enfoques Micro}

- Según la teoría neo-clásica todas elecciones migratorias tienen como única y sola motivación la mejora económica. Sin embargo Conradie y Robeyns comentan el papel de las aspiraciones en el desarrollo humano: "Las aspiraciones pueden jugar al menos dos funciones en las intervenciones de desarrollo humano a pequeña escala: el papel de selección de las capacidades y el papel de la agencia de desbloqueo" (Conradie y Robeyns, 2013) y añaden que las aspiraciones son la esperanza o la ambición de lograr algo en el futuro, incluso sin tener la razonable seguridad de tener éxito, e incluso sin que el objetivo sea el propio bienestar.

- Las teorías neo-clásicas asumen que las preferencias generales en tema de migración son más o menos constantes para todas las personas, en todas las sociedades, durante todos los tiempos, e ignoran que la diferencia de cultura, educación, acceso y exposición a la información puedan tener un gran impacto en el concepto de lo que se considera "buena vida" y la percepción de las oportunidades en otros lugares. El individuo es considerado entonces como un sujeto totalmente abstracto, aislado e independiente del contexto, impermeable a las relaciones sociales y a los valores que estas transmiten, cuando en realidad los migrantes están respaldados por bienes emocionales, financieros, culturales y sociales desiguales que condicionan de forma diferente las expectativas y los deseos de cada persona, su idea de libertad y su capacidad de aprovechar de las oportunidades que le vienen de la vida. Sin embargo unos autores nos advierten que a las aspiraciones hay que añadirles otras características más complejas de medir, como el espíritu emprendedor, la apertura a la innovación, las expectativas de lograr metas valorados, la percepción de nuevas oportunidades o las aspiraciones de movilidad social (De Jong, 2000). 
- Dentro de esta teoría, el comportamiento individual se considera como el resultado totalmente predecible y mecanicista que toma en consideración solo los diferenciales de salario y de oportunidades entre el país de origen y el de acogida, y por lo tanto aunque se hable de la toma de decisiones individual, en realidad no hay un genuino espacio para la agencia.

- Las teorías neo-clásicas no se hacen cargo de los aspectos psicológicos de las personas, mientras que estos entran en juego de manera abrumadora e inequívoca en ocasión de cualquier experiencia migratoria. La capacidad de reorganización interna, las estrategias de aculturación y la capacidad de "coping", así como la creatividad y la complejidad cognitiva son normalmente superiores en los migrantes biculturales que en las personas que solo experimentan o eligen una cultura (LaFromboise, Coleman, y Gerton, 1993).

- Varios estudios que investigan los efectos del proceso de migración a nivel individual están enfocados en el duelo migratorio o en las "fracturas" psicológicas a consecuencia del desplazamiento y de la distancia que pueden haberse generado no sólo entre la sociedad de origen y la de acogida, sino también entre las expectativas del migrante y las condiciones reales. El mensaje que se da por lo tanto es que el problema queda en el individuo y en su incapacidad de integrarse, lo que supone la derivación del sujeto migrante a servicios sociales para que intente solucionar el que viene percatado como su problema, y se le propone la medicalización como respuesta. De esta manera existe el riesgo de que se polarice la atención sólo a los casos problemáticos, sin dar igual reconocimiento y relevancia a todas las experiencias de éxito. Para contrastar esta visión, hay autores como LaFromboise y sus colegas que sugieren que las personas pertenecientes a minorías étnicas que desarrollan las habilidades propuestas en su "Alternation Model" tendrán una mejor salud física y psicológica de las personas que no desarrollaron estas habilidades. Este modelo asume que para una persona es posible conocer, comprender y manejar a la vez dos culturas diferentes y decidir cómo actuar y cómo comportarse según el diferente contexto (LaFromboise y al., 1993).

- La Teoría del Conflicto ha sido criticada por ser excesivamente deductiva y determinista (de Haas, 2011), ya que, aunque tome el individuo como unidad de análisis, en realidad no considera el comportamiento migratorio como expresión de la voluntad individual de la persona, sino que considera el migrante como un peón que se comporta irracionalmente siendo una víctimas pasiva de las macro-fuerzas económicas.

\section{La perspectiva Meso}

Por último, a continuación se enumeran las teorías que profundizan el nivel meso (Faist, 2009), es decir, aquellas teorías que explican el fenómeno tomando en consideración las redes sociales y familiares que condicionan y modifican la migración.

La New Economics of Labour Migration - NELM (Stark y Bloom, 1985) sostiene que la decisión de migrar no la toman sólo los individuos, sino que estos individuos a menudo representan el vehículo de la estrategia familiar para maximizar las posibilidades de ingresos y de supervivencia. La migración es entonces una estrategia colectiva de la red familiar para superar las fallas y deficiencias del mercado, es un intento activo, un acto de agencia por parte de los grupos sociales. 
La Network Theory considera las redes de migrantes como conjuntos de lazos interpersonales que conectan a migrantes, ex migrantes y no migrantes en las zonas de origen y de destino a través de vínculos de parentesco, amistad y pertenencia a la misma comunidad de origen. La red de conexiones constituye una forma de capital social que la gente puede aprovechar para obtener acceso al empleo en el extranjero. La migración se auto-perpetúa y auto-sustenta.

La Migration System Theory destaca la migración como una forma de acción colectiva y una fuerza para la transformación social, que altera las condiciones sociales, culturales, económicas e institucionales tanto en los países emisores, como en los receptores. Con su acción los migrantes pueden obstaculizar el desarrollo de sus países de origen por fuga de cerebros o al contrario facilitarlo a través de las remesas, y a la vez pueden cambiar la composición del trabajo en los países de destino, tanto haciendo posible el perfeccionamiento de nuevos dispositivos de explotación, como también dando nueva vida a los movimientos de revuelta contra ellos.

Para la Teoría Transnacional (Glick Schiller, Basch, y Blanc-Szanton, 1992) los migrantes, lejos de asimilarse invariablemente a las sociedades huéspedes, mantienen relaciones económicas, políticas y sociales con sus lugares de origen. La teoría transnacional abandona el carácter determinista de ruptura permanente, erradicación y abandono de los viejos patrones y de doloroso aprendizaje de nuevos contexto, idioma y cultura, mientras que destaca el nacimiento de un nuevo tipo de población migrante, formada por personas cuyas redes, actividades y estilos de vida incluyen tanto su país de origen como el país de acogida.

\section{Limitaciones que caracterizan los enfoques Meso}

Tomados por si solos, los modelos que consideran la migración como estrategia familiar, asumen que el bienestar de la familia corresponde al bienestar de todos y cada uno de los miembros de la familia. Esto puede ser a nivel teórico, pero en la realidad dentro de la familia pueden ocultarse diferentes intereses y ganancias, acompañados de muy diferentes grados de satisfacción relacionados con la migración. El beneficio de algunos, o inclusive de la unidad familiar en su conjunto, puede ocultar el sacrificio de algunas otras personas, a menudo de las mujeres. Esto quiere decir que para tratar de evaluar el bienestar del individuo debe prestarse mucha atención a la existencia de varios tipos de poder en el ámbito de la familia (Iversen, 2003). Como bien informan los autores feministas, los hogares pueden ser incluso lugares de contención y allí pueden esconderse desequilibrios de poder y fricciones entre los miembros (Killian, Olmsted, y Doyle, 2012).

\section{Una lectura diferente}

En este apartado se propone resumir las limitaciones de las perspectivas tradicionales que se ocupan del proceso migratorio, a través de una clave de lectura específica, es decir subrayando algunas grandes separaciones.

En primer lugar, hay una efectiva separación entre los niveles de análisis (macro, micro, meso) y las disciplinas que se ocupan del problema, lo que no permite abordar el fenómeno de forma integrada. Por lo general, los que tratan las migraciones humanas son economistas, sociólogos y geógrafos, pero cada uno en su campo, sin una colaboración interdisciplinaria, mientras que el estudio se beneficiaría inmensamente si se abordara el tema desde diferentes perspectivas complementarias a la vez (Boyle, Halfacree, y Robinson, 2014).

En segundo lugar, hay una fuerte separación entre las razones económicas y las razones no-económicas. Casi todos los enfoques consideran la migración en función de una 
mejora económica, como si ésta fuera la única o la principal razón que provoca el desplazamiento. Se trata por lo tanto de una perspectiva muy racionalista. En realidad, aunque haya una mejora desde el punto de vista económico, no siempre puede darse por sentado un impacto directo y positivo de la migración en el bienestar general de los migrantes y sus capacidades (de Haas y Rodríguez, 2010) y al revés una mejora de las condiciones de vida no necesariamente pasa por una mejora económica.

Hay que mencionar, además, que hay una fuerte separación entre las restricciones y las oportunidades que conlleva la migración. Las teorías discutidas tratan separar, pesar y medir con valores de signo opuesto, a las que se perciben como oportunidades en el país de destino y a los que se consideraban obstáculos, es decir, condiciones estructurales negativas, en el país de origen. Esto les sirve a los investigadores en la materia para poder creer que tienen bajo control todas las variables involucradas en el proceso. Sin embargo, la experiencia directa de los migrantes muestra que esta separación forzada no representa fielmente la realidad (Briones, 2009).

Podemos condensar lo dicho hasta aquí, comentando que los enfoques macro y micro han sido criticados por su visión determinista, su fragmentación, su racionalismo y su individualismo ontológico (Bonfanti, 2014).

\section{Una propuesta concreta}

Por todas estas razones y con la intención de superar las separaciones anteriormente expuestas, la primera propuesta de este trabajo es la de utilizar el Capability Approach (Sen, 1985) como referencia teórica multidimensional para el examen del bienestar y desarrollo humano de la persona migrante, no solo a partir del aspecto económico, sino analizando todas las variables que componen su calidad de vida. El punto de partida ha sido la teorización inicial del enfoque de Amartya Sen, que considera las capacidades como unidades básicas para el desarrollo humano, para llegar a sus versiones posteriores elaboradas por Martha Nussbaum (Nussbaum, 2001) e Ingrid Robeyns (Robeyns, 2005b).

Los constituyentes principales de este enfoque son las capacidades y los funcionamientos, donde los funcionamientos son los "ser y hacer" (realizados) de una persona, mientras que las capacidades de una persona son "las diversas combinaciones de funcionamientos que una persona puede lograr. Las capacidades son un conjunto de vectores de funcionamientos potenciales, lo que refleja la libertad de la persona para llevar un tipo de vida u otro" (Sen, 1992). Los funcionamientos de una persona y su conjunto de capacidades están estrechamente relacionados, pero son distintos.

Otro punto fundamental en el enfoque de las capacidades son los factores de conversión, que pueden ser personales (por ejemplo, el metabolismo, la condición física, el sexo, las habilidades de lectura, la inteligencia), sociales (como las políticas públicas, las normas sociales, las prácticas que discriminan, los roles de género, las jerarquías sociales, relaciones de poder) o ambientales (por ejemplo, el clima, ubicación geográfica), y que juegan un papel importante en la conversión de los recursos, los productos básicos (es decir, bienes y servicios) en un conjunto de capacidades potenciales.

Para poder convertir las aspiraciones de moverse en el acto concreto del movimiento, a parte de una combinación de factores políticos, económicos y sociales que constituyen la base que hace realmente posible el ejercicio concreto de las capacidades, se requiere también la actuación de lo que Sen y Robeyns llaman "agency", y que Berry por su parte define como "voluntariness", y que dicho de otra manera son la intencionalidad, la capacidad de acción y el ejercicio de poder del individuo, que permite al sujeto actuar sobre la estructura que influye 
sobre el, y que en este contexto se refiere a la motivación de migrar de los individuos (Berry, 1997). Sen define agency como lo que una persona es libre de hacer y alcanzar en la búsqueda de la realización de las metas o los valores que ella considere como importantes (Sen, 1985).

Resumiendo, el acto de migrar depende fuertemente de los factores estructurales, pero implica también claramente la intencionalidad y voluntariedad del individuo.

Esto quiere decir que se vuelve fundamental abordar el fenómeno no sólo con el análisis estadístico y las técnicas propias de la economía, la geografía o la sociología, sino combinar estos instrumentos con herramientas que permitan explorar la agency y la subjetividad de los migrantes, sus expectativas, su grado de satisfacción después de la experiencia migratoria. Es necesario, entonces, complementar los estudios cuantitativos con un análisis cualitativo que permita revelar comportamientos que pueden parecer irracionales según el análisis económico tradicional y que un análisis cuantitativo rara vez puede capturar (Robeyns, 2005a). Un estudio de este tipo permitiría poner en relación tanto los factores de riesgo (racismo, experiencias de prejuicios y discriminación), como los factores de protección (edad, sexo, educación, pre-aculturación, estado social en el momento de la salida del propio país, motivación a la migración, expectativas, redes sociales, distancia cultural, como idioma, religión, etc) con la percepción y la valoración personal de los involucrados.

Aunque existe alguna investigación empírica cuantitativa sobre el bienestar de los migrantes (Safi, 2010), de momento falta en la literatura una investigación que permita explorar, extrapolar y analizar de forma más profunda la percepción sujetiva del bienestar de la población migrante, y que intente comprender si, en su percepción, tras la experiencia migratoria ha aumentado o disminuido su conjunto de capacidades, si considera haber puesto en marcha recursos y habilidades nuevas y diferentes.

\section{Discusión y conclusiones}

En este trabajo se han analizado varias teorías explicativas presentes en la literatura sobre migración y se han identificado algunas limitaciones, que posteriormente han sido resumidas en algunas grandes separaciones.

Esta lectura nos han mostrado cómo, aun teniendo conciencia de que la migración es un fenómeno muy complejo y que es muy difícil encasillarlo en una sola teoría global que abarque toda la complejidad y la riqueza de la vida social (de Haas, 2011), es menester cuestionar los tradicionales modelos de abordaje y ampliar el debate sobre la migración. Con este propósito se ha identificado el Capability Approach y sus posteriores evoluciones, como enfoque privilegiado para estudiar el desarrollo humano más allá de un punto de vista meramente económico y repensar el tema del bienestar de los migrantes desde una perspectiva pluralista e integrada, teniendo en cuenta las diferentes características personales y los múltiples motivos por los que las personas deciden migrar.

Tomando como referencia las conceptualizaciones formuladas en el enfoque de las capacidades, Erik Allardt (Allardt, 1993) ha operacionalizado un modelo para estudiar - a través de las tres dimensiones Tener, Amar, Ser - el nivel de bienestar sujetivo dentro de una sociedad. Su modelo tripartido ha sido la base para realizar encuestas cuatrienales que indagan el grado de calidad de vida (satisfacción, felicidad y sentido de pertenencia) de la población europea (Eurofond, n.d.). El mensaje central de la citada investigación es que la calidad de vida subjetiva, como proveedor principal del bienestar, puede aumentar si se incrementan el nivel de vida de los ciudadanos, mediante el desarrollo de la cultura política y democrática en un país, y se les ofrece mejores oportunidades de vivir su vida de acuerdo con sus necesidades y aspiraciones (Böhnke, 2005). 
Estos estudios consideran la población europea en su totalidad, incluyendo a los migrantes residentes, pero sin una mirada específica sobre este colectivo. El discurso cotidiano tiende a asociar la migración con un incremento del bienestar y supone que los migrantes son (o deberían ser) más feliz por haber logrado migrar hacia el mundo desarrollado. De hecho hay una interesante línea de investigación que se ocupa de la relación entre la migración y las aspiraciones de la población migrante (Czaika \& Vothknecht, 2014). Por otro lado, muchas manifestaciones recientes en Europa han expresado las profundas frustraciones y descontento de los migrantes y sus descendientes.

De todas formas, aunque existe alguna investigación empírica cuantitativa sobre el bienestar de los migrantes (Safi, 2010), falta en la literatura una investigación empírica, que permita explorar, extrapolar y analizar de forma articulada la percepción subjetiva del bienestar de un grupo específico de personas entre la población migrante. Utilizando técnicas narrativas, sería posible recaudar valiosas informaciones con respeto a su calidad de vida en el país de llegada, para comprender si, en su percepción, tras la experiencia migratoria ha aumentado o disminuido su conjunto de capacidades, o si considera haber puesto en marcha recursos y habilidades nuevas y diferentes.

Una visión integrada y sensible a la experiencia subjetiva es necesaria para poder considerar el fenómeno de la migración no sólo como proceso reactivo, problemático y amenazador, sino como un proceso complejo, multidimensional y dinámico, que permite dar un paso fundamental en el proceso de cambio de nuestra colectividad, hacia la idea de una sociedad más inclusiva, enriquecedora, avanzada y justa.

\section{Referencias bibliográficas}

Allardt, E. (1993). Having, Loving, Being: An Alternative to the Swedish Model of Welfare Research. In M. Nussbaum \& A. Sen (Eds.), The Quality of Life (pp. 88-94). Oxford University Press. Retrieved from http://www.oxfordscholarship.com/view/10.1093/0198287976.001.0001/acprof9780198287971-chapter-8

Berry, J. W. (1997). Immigration, Acculturation, and Adaptation. Applied Psychology, 46(1), 5-34. https://doi.org/10.1111/j.1464-0597.1997.tb01087.x

Böhnke, P. (2005). First European Quality of Life Survey: Life satisfaction, happiness and sense of belonging (No. EF0591) (p. 102). Luxembourg: European Foundation for the Improvement of Living and Working Conditions. Retrieved from http://www.eurofound.europa.eu/publications/report/2005/quality-of-life/firsteuropean-quality-of-life-survey-life-satisfaction-happiness-and-sense-of-belonging

Bonfanti, S. (2014). Towards a Migrant-Centred Perspective on International Migration: The Contribution of Amartya Sen's Capability Approach. Social Work \& Society, 12(2), $1-13$.

Boyle, P., Halfacree, K. H., y Robinson, V. (2014). Exploring Contemporary Migration. Routledge.

Briones, L. (2009). Reconsidering the migration-development link: capability and livelihood in Filipina experiences of domestic work in Paris. Population, Space and Place, 15(2), 133-145. https://doi.org/10.1002/psp.532 
Conradie, I., y Robeyns, I. (2013). Aspirations and Human Development Interventions. Journal of Human Development and Capabilities, 14(4), 559-580. https://doi.org/10.1080/19452829.2013.827637

Czaika, M., y Vothknecht, M. (2014). Migration and aspirations - are migrants trapped on a hedonic treadmill? IZA Journal of Migration, 3(1), 1. https://doi.org/10.1186/21939039-3-1

De Haas, H. (2011). The determinants of international migration. Conceptualizing policy, origin and destination effects. IMI Working Papers Series, Paper 32.

De Haas, H., y Rodríguez, F. (2010). Mobility and Human Development: Introduction. Journal of Human Development and Capabilities, 11(2), 177-184. https://doi.org/10.1080/19452821003696798

De Jong, G. F. (2000). Expectations, gender, and norms in migration decision-making. Population Studies, 54(3), 307-319. https://doi.org/10.1080/713779089

Department of Economic and Social Affairs. (2016). International Migration Report 2015: Highlights (Population Division No. ST/ESA/SER.A/375). New York: United Nations.

Eurofond. (n.d.). Encuesta europea sobre calidad de vida (EQLS). Retrieved October 15, 2016, from http://www.eurofound.europa.eu/es/surveys/european-quality-of-lifesurveys

Faist, T. (2009). The Crucial Meso-level. In M. Martiniello \& J. Rath (Eds.), Selected Studies in International Migration and immigration Incorporation. Retrieved from http://pub.uni-bielefeld.de/publication/2604843

Glick Schiller, N., Basch, L., y Blanc-Szanton, C. (1992). Towards a Definition of Transnationalism. Annals of the New York Academy of Sciences, 645(1), ix-xiv. https://doi.org/10.1111/j.1749-6632.1992.tb33482.x

Iversen, V. (2003). INTRA-HOUSEHOLD INEQUALITY: A CHALLENGE FOR THE CAPABILITY APPROACH? Feminist Economics, 9(2-3), 93-115. https://doi.org/10.1080/1354570032000080868

Killian, C., Olmsted, J., y Doyle, A. (2012). Motivated migrants: (Re)framing Arab women's experiences. Women's Studies International Forum, 35(6), 432-446. https://doi.org/10.1016/j.wsif.2012.09.006

LaFromboise, T., Coleman, H., y Gerton, J. (1993). Psychological impact of biculturalism: evidence and theory. Psychological Bulletin, 114(3), 395-412. https://doi.org/10.1037/0033-2909.114.3.395

Martin, P. (2013). The Global Challenge of Managing Migration. Population Bulletin, 68(2).

Nussbaum, M. C. (2001). Women and Human Development: The Capabilities Approach. Cambridge University Press. 
Piore, M. J. (1972). Notes for a theory of labor market stratification (Working Paper). [Cambridge, Mass., M.I.T.]. $\quad$ Retrieved from http://dspace.mit.edu/handle/1721.1/64001

Robeyns, I. (2005a). Selecting Capabilities for Quality of Life Measurement. Social Indicators Research, 74(1), 191-215. https://doi.org/10.1007/s11205-005-6524-1

Robeyns, I. (2005b). The Capability Approach: a theoretical survey. Journal of Human Development, 6(1), 93-117. https://doi.org/10.1080/146498805200034266

Safi, M. (2010). Immigrants' Life Satisfaction in Europe: Between Assimilation and Discrimination. European Sociological Review, 26(2), 159-176. https://doi.org/10.1093/esr/jcp013

Sen, A. (1985). Well-Being, Agency and Freedom: The Dewey Lectures 1984. The Journal of Philosophy, 82(4), 169-221. https://doi.org/10.2307/2026184

Stark, O., y Bloom, D. (1985). The New Economics of Labor Migration. The American Economic Review, 75(2), 173-178.

Todaro, M. P. (1969). A Model of Labor Migration and Urban Unemployment in Less Developed Countries. The American Economic Review, 59(1), 138-148.

Wallerstein, I. (1980). The modern world system. Academic Press. 